Und es wirkt doch!

\section{Yoga lindert chronischen Kreuzschmerz}

Yoga kann als Therapiebaustein bei der Behandlung von Patienten mit chronischem Kreuzschmerz empfohlen werden, so Holger Cramer und Kollegen von der Klinik für Naturheilkunde und Integrative Medizin der Universität Duisburg-Essen. Sie sichteten randomisierte Studien mit Rückenschmerzpatienten aus den Jahren 2004 bis 2011, in denen jeweils eine Yoga-Gruppe mit mindestens einer Kontrollgruppe verglichen wurde. Die Kontrollprobanden erhielten die Standardbehandlung, Rückenübungen oder schriftliche Anweisungen für die Durchführung in Eigenregie. In den Interventionsgruppen der verschiedenen Studien kamen unterschiedliche Yogaarten nach Anleitung zum Einsatz (z.B. Hatha-Yoga, Vini-Yoga, Iyengar-Yoga, meist 1-2 Stunden/ Woche, in bis zu 24 Wochen). Das Maß für den Erfolg war die Effektstärke in Form der standardisierten Mittelwertsdifferenz (SMD) zwischen den Gruppen (Kurzzeiteffekt nach 1-24 Wochen; Langzeiteffekt nach 36 Wochen bis 12 Monaten; Auswertung nach Cohen).

\section{Schmerz signifikant verbessert}

Beim Vergleich mit den Kontrollen zeigte sich bei den Probanden der Yogagruppe direkt nach Beendigung der Intervention eine signifikante Verbesserung beim Schmerz (SMD -0,48) sowie bei den Aktivitätseinschränkungen infolge der Rückenprobleme (SMD -0,59). Für eine Steigerung der Lebensqualität fanden die Autoren keinen Beleg. Dennoch verspürten im Vergleich zur Kontrollgruppe rund dreimal so viele Probanden der Yogagruppen eine allgemeine Verbesserung. Im Langzeitverlauf fiel die Evidenz geringer aus, war aber dennoch bei der Schmerzreduktion (SMD -0,33) und bei der Verbesserung rückenschmerzspezifischer Einschränkungen (SMD -0,35) signifikant. $\mathrm{Zu}$ schwerwiegenden Gesundheitsproblemen kam es bei den Yogaübungen nicht.

Frauen aufgepasst!

\title{
Hoher BMI, hohes Thromboserisiko
}

Übergewichtige Frauen sind stärker gefährdet an einer tiefen Beinvenenthrombose zu erkranken als Normalgewichtige. $\mathrm{Zu}$ dem Ergebnis kam eine englische Studie, die Daten der "Million Woman Study“ auswertete. Bei diesem Screeningprogramm des Nationalen Gesundheitsdienstes von England und Schottland wurden 1.170.495 Frauen mit einem durchschnittlichen Alter von 56 Jahren sechs Jahre lang beobachtet. Frauen mit einem BMI
$>35 \mathrm{~kg} / \mathrm{m}^{2}$ hatten hier im Vergleich zu normalgewichtigen Frauen ein drei- bis vierfach erhöhtes Risiko einer Beinvenenthrombose. Im gleichen Ausmaß traf das auch für Lungenembolien zu. Kam noch eine Operation ins Spiel hatten Frauen 48\% bzw. 78\% häufiger Venenthrombosen, wenn sie übergewichtig bzw. adipös waren.

Parkin L, Sweetland S, Balkwill A et al,
Circulation 2012, 125(15):1897-904

\section{KOMMENTAR}

\section{Von Prof. Dr. Alfred Wirth, Bad Rothenfelde \\ Das viele Fett drückt auf die Venen}

In den letzten Jahren konnten mehrere Untersuchungen überzeugend darlegen, dass nicht nur das arterielle, sondern auch das venöse Gefäßbett mit zunehmendem BMI einem erhöhten Krankheitsrisiko ausgesetzt ist. Große Kohortenstudien sowie eine Metaanalyse weisen aus, dass sowohl eine Beinvenenthrombose als auch eine Lungenembolie bei adipösen Menschen dreimal häufiger vorkommt. Die vorliegende Untersuchung zeigt nun, dass Übergewichtige und Adipöse zusätzlich deutlich häufiger postoperative venöse Komplikationen haben als Normalgewichtige. In anderen Studien wurde gezeigt, dass auch das postthrombotische Syndrom häufiger vorkommt und die Antikoagulation schwieriger ist. Aus diesen Ergebnissen lassen sich folgende Schlussfolgerungen ziehen:

- Ein hoher BMI erhöht das Risiko für eine Venenthrombose.

- Ein hoher BMI bedingt häufiger Operationen, da die operativ zu behandelnden Krankheiten häufig mit Adipositas assoziiert sind (z.B. Cholezystolithiasis, degenerativeWirbelsäulen-und Gelenkerkrankungen, einige gynäkologische Krankheiten, manche Malignome).
Wird operiert, tritt bei hohem BMI häufiger postoperativ eine Venenthrombose auf.

Akute Venenthrombosen gehen bei Adipösen häufiger in ein postthrombotisches Syndrom über.

- Die Antikoagulation ist aus verschiedenen Gründen bei Adipösen häufiger unzureichend.

Die zugrundeliegenden Mechanismen für die Entstehung einer Venenthrombose bei vermehrter Körperfettmasse sind nicht ganz geklärt. Einfach zu verstehen ist, dass vermehrtes intraabdominales Fett die großen Bauchvenen komprimiert, damit den Druck in distalen Venen erhöht und deren Blutfluss reduziert. Adipöse haben im Mittel höhere Plasmakonzentrationen von Fibrinogen sowie einigen Gerinnungsfaktoren, auch die fibrinolytische Aktivität ist reduziert. Einige Produkte des Fettgewebes (Adipokine) sind inflammatorisch wirksam (z.B. TNF-alfa, Interleukine), was die Thrombosebereitschaft ebenfalls erhöht.

Info Diabetologie 2013, 7 (1): 32 